\title{
Prevalencia del uso de medicina tradicional herbolaria $y$ el perfil de uso en pacientes con diabetes tipo 2 de una zona urbana.
}

Milena Abigail Reyes Castro ${ }^{1}$ (D), Leticia Blanco Castillo ${ }^{2}$ iD, Liliana Galicia Rodríguez ${ }^{3}$ iD Emma Rosa Vargas Daza ${ }^{3}$ iD, Enrique Villarreal Ríos ${ }^{3}$

\footnotetext{
${ }^{1}$ Instituto Mexicano del Seguro Social, Unidad de Medicina Familiar No 9, Querétaro, México.

${ }^{2}$ Instituto Mexicano del Seguro Social, Coordinación Clínica de Educación e Investigación, Unidad de Medicina Familiar No 9, Querétaro, México.

${ }^{3}$ Instituto Mexicano del Seguro Social, Unidad de Investigación Epidemiológica y en Servicios de Salud Querétaro, México.
}

Cómo referenciar este artículo/ How to reference this article:

\begin{abstract}
Reyes Castro MA, Blanco Castillo L, Galicia Rodríguez L, Vargas Daza ER, Villarreal Ríos E. Prevalencia del uso de medicina tradicional herbolaria y el perfil de uso en pacientes con diabetes tipo 2 de una zona urbana. Mem. Inst. Investig. Cienc. Salud. 2021; 19(3): 73-82.
\end{abstract}

\section{RE S U M E N}

En México la interculturalidad en salud se plantea como una integración de dos sistemas de conocimiento, el conocimiento ancestral carente de bases científicas y que se transmite a través de las generaciones, y el modelo médico convencional. En múltiples estudios se describe que existe un gran porcentaje de médicos que han recibido pacientes que hacen uso de esta terapéutica. El objetivo fue identificar la prevalencia del uso de medicina tradicional herbolaria y el perfil de uso en pacientes con diabetes tipo 2 en una zona urbana. Se realizó un estudio observacional, transversal descriptivo en pacientes con diagnóstico de diabetes mellitus tipo 2, pertenecientes a un sistema de seguridad social de la ciudad de Querétaro, en un periodo mayor a 6 meses. El plan de análisis estadístico incluyó promedios, porcentajes, intervalos de confianza para promedios e intervalos de confianza para porcentajes. Se identificó que la prevalencia de uso de la medicina tradicional herbolaria era de $22.2 \%$, con una edad promedio de 60.98 años, con predominio en el sexo femenino y una escolaridad secundaria o menos, la planta más utilizada fue moringa en un $45 \%$, el uso más frecuente para el control glucémico en un $97 \%$ y su consumo era como agua de tiempo en la mayoría de las plantas. En este estudio se pudo describir la prevalencia de uso en una zona urbana, así como el perfil de las personas que hacen uso de esta terapéutica, con la finalidad de generar nuevas informaciones y promover el estudio de estas prácticas.

Palabras clave: Interculturalidad, Diabetes tipo 2, Medicina herbaria, Medicina tradicional, Plantas medicinales, Moringa.

\section{Prevalence of the use of traditional herbal medicine and the profile for the use in patients with diabetes type 2 in an urban zone.}

\section{A B S T R A C T}

In Mexico the interculturality in health is posed as an integration of the two systems of knowledge, the ancient knowledge lacking scientific bases which is transmitted Fecha de recepción: octubre 2021. Fecha de aceptación: noviembre 2021 *Autor correspondiente: Milena Abigail Reyes Castro. Dirección Postal: Guadalupe Victoria 100, Felipe Carrillo Puerto, CP.76138 Santiago de Querétaro, Qro. México. Email: drareyes15@gmail.com 
through generations, and the conventional medical model. In multiple studies, the existence of a great percentage of medical doctors that have received patients using this therapy is described. The objective was to identify the prevalence of the use of herbal traditional medicine and the profile of use in patients with diabetes type 2 in an urban zone. This was an observational, transversal descriptive study performed in patients with diagnostic of diabetes mellitus type 2, belonging to a system of social security in Queretaro city, in a period greater than 6 months. The plan of statistical analysis included averages, percentages, confidence intervals for averages and percentages. The prevalence of use of the herbal traditional medicine was $22.2 \%$ with and average age of 60.98 years old, with predominance of the female sex and a scholarship of middle school or less, the plant mostly used was moringa in a $45 \%$, the most frequent use was the glycemic control in $97 \%$ and the consumption was as natural water in most plants. In this study, it was described the prevalence of the use in an urban zone, as well as the profile of the people that use this therapy, with the purpose of generate new information and promote the study of these practices.

Keywords: Interculturality, Diabetes Type 2, Herbal Medicine, Traditional Medicine, Medicinal Plants, Moringa.

\section{INTRODUCCIÓN}

Los usos y costumbres de las personas, así como sus procesos de interrelación y comunicación de saberes se definen desde la década de 1950 como Interculturalidad. Según la Real Academia Española la interculturalidad se refiere a la relación entre diferentes culturas ${ }^{(1,2)}$.

El término se han venido acuñando y promulgando por instituciones como la Organización de las Naciones Unidas para la Educación, la Ciencia y la Cultura (UNESCO), Organización Mundial de la Salud (OMS) y la Organización Panamericana de la salud (OPS), entre otras, quienes con la aprobación de la política sobre etnicidad y salud, reconocen la importancia de adoptar un enfoque intercultural para abordar las inequidades en la salud, generando la interculturalidad en salud, la convergencia de dos sistemas de conocimiento integrado por la medicina académica, también denominada medicina alópata y la medicina tradicional, la cual está basada en conocimientos ancestrales ${ }^{(3-5)}$.

El constante objetivo de esta interculturalidad en salud de acuerdo a la OMS 2014, se enfoca en aprovechar la contribución de la medicina tradicional en el bienestar y atención de la salud. Ya que el reconocimiento de los conocimientos ancestrales es esencial para aprovechar el potencial de la medicina tradicional y contribuir al acceso y cobertura universal en salud, en donde se encuentren incluidos los servicios nacionales de salud y la adopción de intervenciones para el autocuidado desde una perspectiva intercultural ${ }^{(6,7)}$.

Las enfermedades crónicas son un problema de salud pública que cada día se encuentran más al alza y que comprenden una serie de factores que determinan su presencia; dentro de ellas se encuentra la diabetes, la cual año con año eleva su prevalencia dentro de la población mundial, tan solo en 2019 se reportaron 463 millones de pacientes con diabetes tipo 2 a nivel mundial ${ }^{(8-10)}$.

El paciente con diabetes requiere un tratamiento multidisciplinario por la afectación que presenta a todos los niveles, y en la mayoría de las ocasiones esto lleva a una prescripción excesiva de medicamentos por parte del médico 0 a una automedicación $^{(11-13)}$. Por lo que el uso de tratamientos alternativos es una realidad, dicho en otras palabras, el consumo de estas terapéuticas alternativas es una manifestación de interculturalidad ${ }^{(14-16)}$.

En algunos de los servicios de atención a la salud se ha visto la importancia de tomar en cuenta los usos y costumbres de las personas en el manejo y control de la diabetes, para lograr no solo una mejor comunicación médico paciente, sino además su integración para el logro de las metas terapéuticas ${ }^{(17,18)}$. Este enfoque de interculturalidad en salud tiene como finalidad aprovechar la contribución de la medicina tradicional herbolaria en el bienestar y atención de la salud ${ }^{(19-21)}$. 
El objetivo del presente artículo es determinar la prevalencia del uso de medicina tradicional herbolaria en pacientes con diabetes tipo 2, así como su perfil de uso.

\section{MATERIAL Y MÉTODOS}

Se captó a la población blanco que se encontraba en la sala de espera de la unidad de medicina familiar, para integrarla se le preguntó directamente al paciente si padecía o no diabetes, si contestaban afirmativamente se invitaba a participar en el estudio de investigación. Se hizo uso de un consultorio en donde se brindó un ambiente de seguridad y confianza, se realizó entrega del consentimiento informado para que contaran por escrito con la información previamente explicada y su derecho a retirarse o quedarse, así como los beneficios de esta investigación a mediano o largo plazo. Se procedió a la firma para comenzar con la aplicación del instrumento U plantmed (índice de Cronbach de $0.8^{(22)}$, el cuál es el primer instrumento diseñado para identificar el uso de plantas medicinales y las aplicaciones terapéuticas, el cual cuenta con el tiempo aproximado de entrevista fue de 15 minutos por paciente y al término se agradeció su participación en el estudio.

Se recabaron los datos por medio de los datos proporcionados por los pacientes y el registro en expediente electrónico. Se realizó un muestreo no probabilístico por casos consecutivos, empleando como marco muestral los pacientes que acudieron a solicitar atención en la Unidad Médica. Se integraron todos los resultados en una base de datos, en donde se realizó el análisis para posteriormente reportar los resultados.

Se realizó un estudio transversal descriptivo en pacientes con diagnóstico de diabetes mellitus tipo 2, pertenecientes a un sistema de seguridad social de la ciudad de Querétaro, México.

Se calcularon dos tamaños de muestra el primero para definir la prevalencia de uso de medicina tradicional herbolaria y el segundo para identificar las características de uso de la medicina tradicional, en total se realizaron 500 encuestas a pacientes. Para la prevalencia de uso se utilizó la fórmula de porcentajes para población infinita con nivel de confianza del 95\% (Zalfa $=1.64)$, asumiendo que la prevalencia de uso de medicina tradicional herbolaria era de $80 \%(P=0.80)$, y margen de error de $5 \%$ $(d=0.05)$, con el tamaño estimado $168(n=168)$.

El segundo tamaño de muestra se calculó para identificar el perfil de las características de uso de la medicina tradicional herbolaria, en este caso se trabajó con la fórmula de porcentajes para una población infinita con nivel de confianza del $95 \%$ (alfa $=1.64)$, asumiendo que la parte de la planta consumida corresponde a la hoja en el $50 \%$ de los casos $(P=0.50)$ y margen de error de $5 \%(d=0.05)$, el tamaño de muestra calculado fue $268(n=268)$.

La técnica muestral fue no aleatoria por casos consecutivos, empleando como marco muestral el listado de pacientes que acudieron a la unidad de salud a solicitar atención médica.

Se estudiaron variables sociodemográficas (edad, sexo, escolaridad (secundaria o menos) y religión. El uso de medicina tradicional y perfil de uso, operacionalizado a través de instrumento $\mathrm{U}$ - PlantMed, el cual consta de las siguientes 6 preguntas ¿Usa medicina tradicional? ¿Qué tipo de planta utiliza?, ¿Con que finalidad usa la medicina tradicional?, ¿Qué parte de la planta utiliza?, ¿Cómo prepara la planta?, ¿Qué dosis usa?

En el tratamiento médico farmacológico, se identificó el tipo de medicamento prescrito por el médico tratante y la cantidad de tabletas consumidas al día en los últimos 6 meses. Las características de salud incluyeron IMC (peso y talla) perímetro de cintura; cifras de presión arterial sistólica y diastólica; las características bioquímicas: la glucosa, colesterol y triglicéridos; control metabólico para la glucosa. $(120 \mathrm{mg} / \mathrm{dl})$, colesterol $(200 \mathrm{mg} / \mathrm{dl})$, triglicéridos $(150 \mathrm{mg} / \mathrm{dl})$ TA sistólica $(130 \mathrm{~mm} \mathrm{hg})$ y TA diastólica ( $90 \mathrm{~mm} \mathrm{hg}$ ) los cuales se obtuvieron del expediente electrónico.

El plan de análisis estadístico incluyó promedios, porcentajes, intervalos de confianza para promedios e intervalos de confianza para porcentajes. 


\section{RESULTADOS}

La prevalencia del uso de la medicina tradicional herbolaria es $22.2 \%$ (IC $95 \%$; 18.7-25.7).

La edad promedio de la población que consume plantas medicinales es 60.98 años (IC 95\%; 58.57-63.04), predomina el sexo femenino con 59.6\% (IC 95\%; 46.6-70.6), la escolaridad secundaria o menos está presente en $64.4 \%$ (IC 95\%; 51.8-76.9) y la religión predominante es la católica con $86.4 \%$ (IC 95\%; 77.4-95.2).

El medicamento más utilizado es metformina con $53.5 \%$ y el promedio de tabletas por día es 2.03. En la Tabla 1 se presenta la prevalencia por tipo de medicamento y tabletas consumidas por día.

El promedio de glucosa en los pacientes con diabetes tipo 2 que usan medicina tradicional herbolaria es $170.98 \mathrm{mg} / \mathrm{dl}$ (IC 95\%; 151.37-190.59) y se encuentran controlados $33.8 \%$ (IC 95\%; 21.4-46.3). En la Tabla 2 se presentan las características físicas, clínicas, bioquímicas y el porcentaje de control metabólico.

La planta más utilizada en pacientes con diabetes tipo 2, fue la moringa (Moringa oleífera) con 45.0\% (IC 95\%; 36.4-53.6), en segundo lugar, la planta de insulina (Boussingoltiabasselloides) con 17.1\% (IC 95\%; 10.6- 23.6). En la Tabla 3 se presenta la prevalencia por tipo de planta.

La parte de la planta que más se utilizaba eran las hojas como en el caso de la moringa (Moringa oleifera) $55.2 \%$, esta parte fue también la más utilizada en la planta de insulina(Boussingoltiabasselloides) con $77.3 \%$ y en la hoja de higo (Ficus carica) en el $100 \%$ de los casos. En la tabla IV se presenta por tipo de planta la parte utilizada.

La moringa (Moringa oleifera) se utiliza para el control, glucémico en $97.0 \%$ de los casos y para relajación en $3.0 \%$. En el resto de las plantas, la finalidad era el control glucémico en todos los casos.

El tipo de preparación más común fue en forma de té, el cual consiste en hervir la planta medicinal con la finalidad de extraer sus propiedades, usado en el jengibre (Zingiberofficinale) y ginseng (Panaxgingseng) en el $100 \%$ de los casos, $69.0 \%$ en la moringa (Moringa oleifera), $68.2 \%$ en la planta de insulina (Boussingoltiabasselloides), $36.4 \%$ en el nopal (Opuntia vulgaris) y $31.3 \%$ en el ajo (Alliumsativum). En la Tabla 4 se presenta la prevalencia del tipo de preparación por planta.

El consumo más común era como agua de tiempo en la mayoría de las plantas, el cual consiste en la toma de té o infusiones a temperatura ambiente, sustituyendo el agua que se consume a lo largo del día por la preparación. En la Tabla 5 se presenta la prevalencia por tipo de uso.

Tabla 1. Prevalencia por tipo de medicamento y unidades por día en pacientes con diabetes tipo 2 que hacen uso de la medicina tradicional herbolaria. $n=268$

\begin{tabular}{lccccccc}
\hline Medicamento & $\begin{array}{l}\text { Prevalencia } \\
\text { de uso (\%) }\end{array}$ & \multicolumn{2}{c}{ IC } & $\begin{array}{c}\text { Promedio } \\
\text { unidades } \\
\text { por día }\end{array}$ & \multicolumn{2}{c}{ IC } & \\
& & Inferior & Superior & & Inferior & Superior \\
\hline $\begin{array}{l}\text { Metformina } \\
\text { (tabletas) }\end{array}$ & 53.5 & 47.5 & 59.5 & 2.03 & 0.3 & 3.7 \\
$\begin{array}{l}\text { Insulina } \\
\text { (dosis) }\end{array}$ & 27.9 & 22.5 & 33.3 & 1.67 & 0.1 & 3.2 \\
$\begin{array}{l}\text { Glibenclamida } \\
\text { (tabletas) }\end{array}$ & 14 & 9.8 & 18.2 & 2.22 & 0.5 & 4.0 \\
$\begin{array}{l}\text { Pioglitazona } \\
\text { (tabletas) }\end{array}$ & 2.3 & 0.5 & 4.1 & 2.00 & 0.3 & 3.7 \\
$\begin{array}{l}\text { Acarbosa } \\
\text { (tabletas) }\end{array}$ & 2.3 & 0.5 & 4.1 & 2.00 & 0.3 & 3.7 \\
\hline
\end{tabular}


Tabla 2. Características físicas, clínicas, bioquímicas y control metabólico en pacientes con diabetes tipo 2 que hacen uso de la medicina tradicional herbolaria. $n=268$

\begin{tabular}{|c|c|c|c|}
\hline \multirow{2}{*}{ Característica } & \multirow{2}{*}{ Promedio } & \multicolumn{2}{|c|}{ IC $95 \% n=268$} \\
\hline & & inferior & Superior \\
\hline \multicolumn{4}{|l|}{ Características físicas } \\
\hline Peso (KG) & 77.74 & 73.82 & 81.66 \\
\hline Talla(CM) & 1.58 & 1.56 & 1.60 \\
\hline IMC & 30.56 & 29.13 & 32.00 \\
\hline Cintura (CM) & 110.66 & 96.75 & 104.56 \\
\hline \multicolumn{4}{|l|}{ Características clínicas } \\
\hline TA Sistólica(mm hg) & 142.83 & 102.72 & 182.94 \\
\hline TA Diastólica(mm hg) & 74.61 & 72.19 & 77.03 \\
\hline \multicolumn{4}{|c|}{ Características bioquímicas } \\
\hline Glucosa (mg/dl) & 170.98 & 151.37 & 190.59 \\
\hline Colesterol (mg/dl) & 188.76 & 177.77 & 199.74 \\
\hline Triglicéridos (mg/dl) & 198.59 & 163.34 & 233.83 \\
\hline Control metabólico \% & & & \\
\hline \multirow{3}{*}{$\begin{array}{l}\text { Glucosa (controlada) } \\
\text { Colesterol } \\
\text { (controlado) }\end{array}$} & 33.8 & 21.4 & 46.3 \\
\hline & & & \\
\hline & 62.7 & 50.0 & 75.4 \\
\hline \multirow{2}{*}{$\begin{array}{l}\text { Triglicéridos (controlado) } \\
\text { Presión arterial } \\
\text { (controlada) }\end{array}$} & 42.3 & 29.3 & 55.3 \\
\hline & 83.0 & 73.1 & 92.9 \\
\hline
\end{tabular}

Tabla 3. Prevalencia por tipo de planta en pacientes con diabetes tipo 2 que hacen uso de la medicina tradicional herbolaria. $n=268$

\begin{tabular}{lccc}
\hline & IC 95\% & $\mathbf{n = 2 6 8}$ \\
\hline Tipo de planta & Porcentaje & Inferior & Superior \\
\hline $\begin{array}{l}\text { Moringa } \\
\text { (Moringa oleifera) }\end{array}$ & 45.0 & 36.4 & 53.6 \\
$\begin{array}{l}\text { Planta de insulina } \\
\text { (Boussingoltiabasselloides }\end{array}$ & 17.1 & 10.6 & 23.6 \\
$\begin{array}{l}\text { Ajo } \\
\text { (Alliviumsativum) }\end{array}$ & 12.4 & 6.7 & 18.1 \\
$\begin{array}{l}\text { Nopal } \\
\text { (Opuntia vulgaris) }\end{array}$ & 8.5 & 3.7 & 13.3 \\
$\begin{array}{l}\text { Hojas de higo } \\
\text { (Ficus carica) }\end{array}$ & 3.1 & 0.1 & 6.1 \\
$\begin{array}{l}\text { Jengibre } \\
\text { (Zingiberofficinale) }\end{array}$ & 3.1 & 0.1 & 6.1 \\
$\begin{array}{l}\text { Ginseng } \\
\text { (Panaxgingseng) }\end{array}$ & 2.3 & 0.0 & 4.9 \\
$\begin{array}{l}\text { (Aloe vera) } \\
\text { Cúrcuma } \\
\text { (Cúrcuma longa) }\end{array}$ & 2.3 & 0.0 & 4.9 \\
$\begin{array}{l}\text { Semilla de Brasil } \\
\text { (Bettholletia excelsa) } \\
\text { Clorofila }\end{array}$ & 2.3 & 0.0 & 4.9 \\
\hline
\end{tabular}


Tabla 4. Partes de la planta consumida en pacientes con diabetes tipo 2 que hacen uso de la medicina tradicional herbolaria. $n=268$

\begin{tabular}{|c|c|c|c|c|c|c|}
\hline Tipo de planta & Hoja \% & Tallo \% & Raíz \% & $\begin{array}{c}\text { Flores } \\
\%\end{array}$ & $\begin{array}{c}\text { Semillas } \\
\%\end{array}$ & $\begin{array}{c}\text { Otras } \\
\%\end{array}$ \\
\hline $\begin{array}{l}\text { Moringa } \\
\text { (Moringa oleifera) }\end{array}$ & 55.2 & 5.2 & 0.0 & 3.4 & 36.2 & 0.0 \\
\hline $\begin{array}{l}\text { Planta de insulina } \\
\text { (Boussingoltiabasselloi } \\
\text { des) }\end{array}$ & 77.3 & 13.6 & 0.0 & 4.5 & 4.5 & 0.0 \\
\hline $\begin{array}{l}\text { Ajo } \\
\text { (Alliviumsativum) }\end{array}$ & 0.0 & 0.0 & 0.0 & 0.0 & 100.0 & 0.0 \\
\hline $\begin{array}{l}\text { Sábila } \\
\text { (Aloe vera) }\end{array}$ & 0.0 & 100.0 & 0.0 & 0.0 & 0.0 & 0.0 \\
\hline $\begin{array}{l}\text { Nopal } \\
\text { (Opuntia vulgaris) }\end{array}$ & 0.0 & 72.7 & 9.1 & 0.0 & 18.2 & 0.0 \\
\hline Clorofila & 0.0 & 0.0 & 0.0 & 0.0 & 0.0 & 100.0 \\
\hline $\begin{array}{l}\text { Cúrcuma } \\
\text { (Cúrcuma longa) }\end{array}$ & 0.0 & 33.3 & 0.0 & 33.3 & 33.3 & 0.0 \\
\hline $\begin{array}{l}\text { Hojas de higo } \\
\text { (Ficus carica) }\end{array}$ & 100.0 & 0.0 & 0.0 & 0.0 & 0.0 & 0.0 \\
\hline $\begin{array}{l}\text { Jengibre } \\
\text { (Zingiberofficinale) }\end{array}$ & 0.0 & 50.0 & 50.0 & 0.0 & 0.0 & 0.0 \\
\hline $\begin{array}{l}\text { Ginseng } \\
\text { (Panaxgingseng) }\end{array}$ & 100.0 & 0.0 & 0.0 & 0.0 & 0.0 & 0.0 \\
\hline $\begin{array}{l}\text { Semilla de Brasil } \\
\text { (Bettholletia excelsa) }\end{array}$ & 50.0 & 0.0 & 0.0 & 0.0 & 50.0 & 0.0 \\
\hline
\end{tabular}

Tabla 5. Tipo de preparación por tipo de planta y tipo de uso en pacientes con diabetes tipo 2 que hacen uso de la medicina tradicional herbolaria. $n=268$

\begin{tabular}{|c|c|c|c|c|c|c|c|c|}
\hline \multirow[b]{2}{*}{ Tipo de planta } & \multicolumn{4}{|c|}{ Tipo de preparación \% } & \multicolumn{4}{|c|}{ Tipo de uso \% } \\
\hline & Té & infusión & polvo & otros & $\begin{array}{c}\text { consumo } \\
\text { como } \\
\text { agua de } \\
\text { tiempo } \\
\end{array}$ & $\begin{array}{c}\text { consu } \\
\text { mo } \\
\text { ocasio } \\
\text { nal }\end{array}$ & $\begin{array}{l}\text { consu } \\
\text { mo } \\
\text { diario }\end{array}$ & $\begin{array}{l}\text { consu } \\
\text { mo en } \\
\text { ayuno }\end{array}$ \\
\hline $\begin{array}{l}\text { Moringa } \\
\text { (Moringa oleifera) }\end{array}$ & 69.0 & 5.2 & 5.2 & 20.7 & 50.0 & 27.6 & 17.2 & 5.2 \\
\hline $\begin{array}{l}\text { (Boussingoltiabas } \\
\text { selloides) }\end{array}$ & 68.2 & 18.2 & 0.0 & 13.6 & 40.9 & 18.2 & 13.6 & 27.3 \\
\hline $\begin{array}{l}\text { Ajo } \\
\text { (Alliviumsativum) }\end{array}$ & 31.3 & 18.8 & 0.0 & 50.0 & 6.3 & 43.8 & 31.3 & 18.8 \\
\hline $\begin{array}{l}\text { Sábila } \\
\text { (Aloe vera) }\end{array}$ & 33.3 & 33.3 & 0.0 & 33.3 & 0.0 & 66.7 & 0.0 & 33.3 \\
\hline $\begin{array}{l}\text { Nopal } \\
\text { (Opuntia vulgaris) }\end{array}$ & 36.4 & 9.1 & 0.0 & 54.5 & 18.2 & 27.3 & 27.3 & 27.3 \\
\hline Clorofila & 0.0 & 0.0 & 0.0 & 100.0 & 100.0 & 0.0 & 0.0 & 0.0 \\
\hline $\begin{array}{l}\text { Cúrcuma } \\
\text { (Cúrcuma longa) }\end{array}$ & 33.3 & 33.3 & 0.0 & 33.3 & 0.0 & 66.7 & 33.3 & 0.0 \\
\hline $\begin{array}{l}\text { Hojas de higo } \\
\text { (Ficus carica) }\end{array}$ & 100.0 & 0.0 & 0.0 & 0.0 & 50.0 & 0.0 & 25.0 & 25.0 \\
\hline $\begin{array}{l}\text { Jengibre } \\
\text { (Zingiberofficinale) }\end{array}$ & 100.0 & 0.0 & 0.0 & 0.0 & 75.0 & 25.0 & 0.0 & 0.0 \\
\hline $\begin{array}{l}\text { Ginseng } \\
\text { (Panaxgingseng) }\end{array}$ & 33.3 & 0.0 & 33.3 & 33.3 & 0.0 & 0.0 & 100.0 & 0.0 \\
\hline $\begin{array}{l}\text { Semilla de Brasil } \\
\text { (Bettholletia } \\
\text { excelsa) }\end{array}$ & 50.0 & 50.0 & 0.0 & 0.0 & 50.0 & 0.0 & 50.0 & 0.0 \\
\hline
\end{tabular}




\section{DISCUSIÓN}

La medicina tradicional se encuentra presente en $22.2 \%$ de la población, como podemos observar es una baja proporción comparado con lo reportado en la literatura a nivel mundial ${ }^{(12,13,18,21)}$, esto puede estar originado por el éxito de los programas educativos dirigidos a la población con diabetes, en los cuales se abordan los riesgos del consumo de este tipo de tratamientos tradicionales, en donde se destaca la falta de una dosis efectiva o la presencia de interacciones y en donde se les hace énfasis del libre albedrío para su consumo, orientando en todo momento al paciente, si éste desea tomarlo, se aconseja no suspender el uso del tratamiento alópata. Estos cambios en las recomendaciones del personal de salud son guiados por la interculturalidad en salud en donde la visión principal es permitir al individuo continuar con sus usos y costumbres, guiados del conocimiento científico, permitiendo perpetuar su cultura sin poner en riesgo la salud de las personas.

Desde el momento en que el paciente con diabetes llega a la consulta de medicina familiar, el médico debe respetar la concepción del paciente sobre su enfermedad y proporcionarle las herramientas necesarias para que el paciente pueda prevenir o en algunos casos manejar y controlar su padecimiento, así como acompañarlo en el proceso, de ahí la importancia de conocer las alternativas que la medicina tradicional les ofrece y de las cuales hacen uso. En esto radica la importancia del artículo que aquí se presenta.

De los pacientes que consumieron medicina tradicional, el promedio de edad pertenece al grupo de sesenta y más, lo cual concuerda con lo reportado en la literatura existente. Acosta en el 2018 realizo un estudio en la población de Asunción en donde el promedio de edad de pacientes que usaban medicina tradicional herbolaria fue de 62 años, en el mismo año Paniagua realizo un estudio en población mexicana donde el promedio de edad obtenido fue de 60 años, mientras queCuenca ${ }^{(12)}$ en el 2020 al realizar su estudio en Ecuador obtuvo un promedio de edad de 45 a 60 años. Este comportamiento se puede explicar en función de la cultura propia del adulto mayor, el cual creció en un sistema de salud, más orientado hacia las prácticas tradicionales que a la medicina institucional.

Las plantas conocidas con efecto hipoglucemiante conforman un listado de más de $400^{(23)}$, sin embargo algunas son más recomendadas que otras por la cantidad de padecimientos sobre los cuales inciden, dentro de los resultados que se encontraron el uso de la moringa se posiciona en el primer lugar de consumo, la cual es una planta con efecto antibiótico, anorexígeno y metabólico, reconocido por la Organización de las Naciones Unidas para la Alimentación y la Agricultura, es de amplia distribución a nivel mundial y sus condiciones de producción son muy asequibles a cualquier población, es por ello que sea una de las más utilizadas y consumidas a nivel mundial, por otro lado se observó que la planta de insulina es igualmente utilizada en la medicina tradicional por sus efectos hipoglucemiantes, sin embargo con una menor frecuencia de uso; de esta planta llama la atención el nombre, el cual hace referencia a medicamentos alópatas existentes, lo cual puede significar una asociación del efecto terapéutico de la medicina tradicional y la medicina alópata para el paciente.

En las formas de consumo más utilizadas se encontró el té, el cual tiene una connotación diferente a nivel cultural, pues el término de té propiamente hace referencia al uso de la planta china Camelliasinensis, sin embargo, culturalmente se le atribuye el término té a cualquier preparación en la cual se colocan partes de hojas, hierbas, frutos o cortezas las cuales se hierven con la finalidad de extraer sus propiedades y posteriormente se cuela, sin embargo existen otras prácticas como la infusión en donde se deja reposar la planta o hierba en agua caliente durante unos minutos y posteriormente está lista para servirse, desde tiempos ancestrales las costumbres marcan que para extraer todas las propiedades de una planta se debe hervir y dejar reposar, obteniendo un producto más concentrado y es esta práctica la que ha pasado de generación en generación hasta nuestros días, motivo por el cual puede ser la más usada. 
Dentro de los usos y costumbres de la medicina tradicional se encuentra el término agua de uso, el cual hace referencia a la toma de té o infusiones a temperatura ambiente, sustituyendo el agua de consumo por el preparado para su toma a lo largo del día, dentro de los resultados encontrados, observamos que esta usanza prevalece aunque en menor proporción, siendo más popular el consumo ocasional y como complemento al tratamiento alópata, ya que no se mostraron tendencias al abandono del tratamiento, por el contrario, se encontró que los pacientes tienen un consumo promedio de dos tomas del tratamiento alópata al día, al cual se le agrega de manera ocasional el consumo de medicina tradicional con la finalidad de llegar a un control metabólico, el cual en muchas ocasiones no se logra, ya que se encontró que solo un $33.8 \%$ de los consumidores de medicina tradicional presentó control metabólico, en variables físicas, químicas y bioquímicas.

Existe una vasta cultura relacionada con la medicina tradicional, la cual proviene de la época prehispánica y su principal representante es la herbolaria, sobreviviendo a lo largo de los años, adaptándose a las exigencias de la población, la cual está en constante cambio, actualmente se puede acceder a ella en diferentes presentaciones que van desde cápsulas, suplementos, polvo, hasta la misma planta. El paciente con diabetes se encuentra frente a una amplia gama de posibles tratamientos disponibles, que varían de acuerdo a la diversidad de las plantas y la forma de preparación, generando una esperanza de curación, de acuerdo a los relatos e información disponible de estas plantas.

Un gran porcentaje de los individuos en algún momento de la vida han hecho uso de la medicina tradicional de diferentes maneras, en forma de infusiones, té o mediante otras prácticas ya sea por cuenta propia o recomendado por otras personas; proporcionando a la vez un sostén psicológico a los usuarios, ayudando en el proceso de curación de una determinada enfermedad, en este caso la diabetes, siendo un complemento a las recomendaciones y al tratamiento alópata establecido. Popularmente se desconocen las concentraciones idóneas para obtener un efecto terapéutico, lo cual pudiera generar éxito o fracaso en el control metabólico.

Por lo cual podemos concluir que a pesar de que la prevalencia de la medicina tradicional es baja, se encuentra presente en zonas urbanas, tratando de brindar solución a los conflictos y problemas de salud de los pacientes con Diabetes tipo 2.

Conflicto de intereses: Los autores manifiestan no poseer conflicto de interés.

Financiación: Fondos Propios.

Contribución de los autores: Todos los autores han contribuido en la elaboración del presente manuscrito.

\section{REFERENCIAS BIBLIOGRÁFICAS}

1. Real Academia Española (RAE). Diccionario de la lengua española. Madrid: RAE; 2014. Disponible en: https://dle.rae.es/

2. Paulo A, Cruz M. De eso que se ha llamado interculturalidad en salud: un enfoque reflexivo. RevUnivInd Santander Salud [Internet]. 2018 [consultado el 5 de julio del 2021]; 50(4): 366-84.

Doi: $10.18273 /$ revsal.v50n4-2018010

3. Guzmán S. La interculturalidad en salud: espacio de convergencia entre dos sistemas de conocimiento. Rev. Gerenc. Polít. Salud [Internet]. 2016 [consultado el 5 de julio del 2021]; 15 (31): 10-29. Doi:

10.11144/Javeriana.rgyps15-31.isec

4. Guzmán S. Ethnic Minorities and Social Inequities: A diagnostic Assessment about the Mexican Indigenous Population. Open Journal of Social Sciences [Internet]. 2015 [consultado el 5 de julio del 2021] 3(8): 20-34. Doi: 10.4236/jss.2015.38002

5. Gutiérrez G, Espinosa $E$, Hernández $P$, Pavón $T$, Márquez $O$. Instrumento para la medición de la sustentabilidad de la herbolaria. México: 2020 Centro Universitario UAEM Amecameca [sede Web] [acceso 5 julio de 2021]. 
Repositorio institucional. Agrociencia 54 (8): 1043-58. Disponible en: http://hdl.handle.net/20.500.11799/11 $\underline{0254}$

6. Organización Mundial de la Salud. Estrategia de la OMS sobre medicina tradicional 2014-2023 [Internet]. Ginebra (Suiza): OMS; 2013 [consultado el 5 de julio del 2021]. Disponible en: https://apps.who.int/iris/bitstream/han dle/10665/95008/9789243506098 spa. pdf; jsessionid $=9 B B 46187 A 1 A 80237650$ A18487E8EFFE4?sequence $=1$

7. Organización Panamericana de la Salud. Estrategia y plan de acción sobre etnicidad y salud 2019-2025. [Internet] Washington (D.C).: OPS; 2019: [consultado el 5 de julio del 2021]. Disponible en: https://iris.paho.org/handle/10665.2/5 $\underline{1745}$

8. Irigoyen A, Ayala A, Ramírez O, Calzada E. La Diabetes Mellitus y sus implicaciones sociales y clínicas en México y Latinoamérica. Arch en Med Fam. [Internet]. 2017 [consultado el 5 de julio del 2021]; 19(4): 91-94. Disponible en: https://www.medigraphic.com/pdfs/me dfam/amf-2017/amf174c.pdf

9. Mendoza M, Padrón A, Cossío PE, Soria M. Prevalencia mundial de la diabetes mellitus tipo II y su relación con el índice de desarrollo humano. RevPanam Salud Pública. [Internet]. 2017 [consultado el 5 de julio del 2021]; 41(103). Disponible en: Doi: 10.26633/RPSP.2017.103

10.Federación internacional de diabetes. Atlas de la diabetes de la FID novena edición 2019. [Internet]. 2019: [consultado el 5 de julio del 2021]. Disponible en:

https://www.ciberdem.org/noticias/nue vo-atlas-de-la-diabetes-de-la-idf-2019

11. Berenzon S, Ito-Sugiyama E, VargasGuadarrama L. Enfermedades y padeceres por los que se recurre a terapeutas tradicionales de la Ciudad de México. Salud pública de México [Internet]. 2006 [consultado el 5 de julio del 2021]. 48(1). Disponible en: https://www.medigraphic.com/cgibin/new/resumen.cgi?IDARTICULO $=125$ $\underline{89}$

12. Cuenca $P$, Uriarte $M$, Rodríguez J, Parcon M. Uso de la medicina no convencional por pacientes diabéticos. Archmed Camagüey. [Internet]. 2020 [consultado el 5 de julio del 2021]. 24(1): 6632 Disponible en:

http://scielo.sld.cu/scielo.php?script=sci arttext\&pid $=$ S102502552020000100008

13. Paniagua L, Ruiz A, Alfaro E, Ruiz N, Zapata J, Alonso A. Uso de Plantas Medicinales en Pacientes con Diabetes Mellitus II. Ciencias Naturales $Y$ Exactas. [Internet]. 2018 [consultado el 5 de julio del 2021]. 4(1). disponible en:

http://www.jovenesenlaciencia.ugto.mx /index.php/jovenesenlaciencia/article/vi ew/261

14. Sackett K, Carter M, Stanton M. Elders' use of folk medicine and complementary and alternative therapies: an integrative review with implications for case managers. Prof Case Manag [Internet]. 2014 [consultado el 5 de julio del 2021]. 19(3) 113-23; quiz 124-5. Doi: 10.1097/NCM.0000000000000025

15. Maya $P$, Sánchez $C$. De eso que se ha llamado interculturalidad en salud: un enfoque reflexivo. RevUnivInd Santander Salud. 2018; 50(4): 366-84. Doi: $10.18273 /$ revsal.v50n4-2018010

16. Damnjanovic I, Kitic D, Stefanovic N, Zlatkovic-Guberinic S, Catic-Djordjevic A, Velickovic-Radovanovic R. Herbal self-medication use in patients with diabetes mellitus type 2 . Turk J MedSci. 2015; 45(4): 964-71. doi: 10.3906/sag-1410-60.

17. Salaverry O. Interculturalidad en salud. Rev. perú. med. exp. salud publica. [Internet] 2010 [consultado el 5 de julio del 2021] 27(1): 80-93. Disponible en: http://www.scielo.org.pe/scielo.php?scri pt=sci abstract\&pid=S172646342010000100013

18. Acosta P, Lugo G, Vera Z, Morinigo M, Maidana GM, Samaniego L. Uso de plantas medicinales y fitoterápicos en pacientes con Diabetes Mellitus tipo 2. Mem. Inst. Investig. Cienc. Salud. [Internet] 2018 [consultado el 5 de julio del 2021]. 16(2): 6-11. Disponible en: Doi: $\quad 10.18004 / M e m . i i c s / 1812-$ 9528/2018.016(02)06-011

19. Eddouks M, Bidi A, El Bouhali B, Hajji L, Zeggwagh NA. Antidiabetic plants improving insulin sensitivity. J PharmPharmacol [Internet]. 2014 [consultado el 5 de julio del 2021]. 66(9): 1197-214. Disponible en: doi: 10.1111/jphp.12243. Epub $2014 \mathrm{Apr}$ 15. PMID: 24730446. 
20. Veliz $L$, Bianchetti $A$, Silva $M$. Competencias interculturales en la atención primaria de salud: un desafío para la educación superior frente a contextos de diversidad cultural. ESSAY. Cad. Saúde Pública. [Internet]. 2019 [consultado el 5 de julio del 2021] 35(1). Disponible en: https://doi.org/10.1590/0102311X00120818

21. Maidana M, González $Y$, Degen R. Plantas medicinales empleadas por pacientes diabéticos en Paraguay. Rev. Infarma [Internet]. 2015 [consultado el 10 de julio del 2020]; 27(4); 214-20. Disponible en:

http://oaji.net/articles/2016/34251469798399.pdf
22. Gallegos M, Mazacon B, Troncoso L. Diseño y validación del cuestionario $U$ Plan Med para identificación del uso de plantas medicinales en Babahoyo, Ecuador. AnFacmed [Internet]. 2016 [citado 5 de julio de 2021]; 77(3): 2072012. Disponible en: https://revistasinvestigacion.unmsm.ed u.pe/index.php/anales/article/view/123 $\underline{99}$

23. Gallego C, Ferreira F. Plantas medicinales en el tratamiento de la Diabetes Mellitus Tipo 2: una revisión. Farmacéuticos Comunitarios. [Internet]. 2015 [citado 5 de julio de 2021]. 7(4): 27-34. doi: 10.5672/FC.21739218.(2015/Vol7).004.05 